
https://doi.org/10.30534/ijeter/2020/33822020

\title{
Indoor Positioning System Using BLE for Tracking Dynamic User Positions
}

\author{
Christian Alvin Setiabudi ${ }^{1}$, Alvin Riady ${ }^{2}$, Gede Putra Kusuma ${ }^{3}$ \\ ${ }^{1}$ Computer Science Department, BINUS Graduate Program - Master of Computer Science, Bina Nusantara \\ University, Jakarta, Indonesia, 11480, christian.setiabudi@binus.ac.id \\ ${ }^{2}$ Computer Science Department, BINUS Graduate Program - Master of Computer Science, Bina Nusantara \\ University, Jakarta, Indonesia, 11480, alvin.riady@ binus.ac.id \\ ${ }^{3}$ Computer Science Department, BINUS Graduate Program - Master of Computer Science, Bina Nusantara \\ University, Jakarta, Indonesia, 11480, inegara@ binus.edu
}

\begin{abstract}
Indoor Positioning System has been one of the most attractive research after Bluetooth Low Energy (BLE) was introduced. This technology mainly used because of the reduction of material and energy cost over time that has huge impact compared to other technology which are more costly. Most Recent Research has solved the problem of inconsistency on Received Signal Strength (RSS) using fingerprint method. But the RSS that received may contain some noise. This paper mainly proposed a method to estimate or tracking the real position of dynamic user. With RSS value as input to be processed and the result of it will be a location $(\mathrm{x}, \mathrm{y})$ value, then repeat the process to create an estimate coordinate map of route taken. Our proposed method is based on fingerprinting with weighted sum of five nearest reference point to estimate the position of dynamic user then using Extended Kalman Filter as a tracking algorithm. In this paper we try new ways to collect the data of RSS by dynamically collecting the data in many routes to see whether the proposed algorithm could estimate the position better. We achieve an average mean of error around 302,42 cm using Weighted Sum + Extended Kalman Filter tested on dynamic data.
\end{abstract}

Key words: Indoor Positioning System, Dynamic User Localization, Fingerprinting Method, Weighted Sum Model, Extended Kalman Filter.

\section{INTRODUCTION}

Location detection has been popular implemented in outdoor environments by the introduction of GPS technology. GPS has made many changes on our daily activities, the usefulness to navigate to other places has been a very important factor of the success of GPS. Different from outdoor positioning, GPS technology can't be used for indoor positioning. GPS technology had limited access to indoor positioning because of many factors such as lack of line of sight and in indoor the signal of the GPS will be attenuated as they cross through walls [4].
There is many research that is focused on Indoor Positioning System (IPS) until now. The system is capable of diverse purposes such as smart homes, care centres, shopping mall advertisement, etc. The main problematic in this current topic are in Indoor Positioning there is no significant environment that could be applied to every single of Indoor Positioning, simply, there is no standardization about a building structure and in other case the indoor positioning system would behave differently. Also, in Indoor positioning there is many obstacle that need to be included as an obstruction. This cause the GPS could not applied into IPS.

Various indoor positioning technologies has been proposed based on the literature review such as Infrared, Ultrasound, WLAN/Wi-Fi, BLE and RFID to estimate the position of user in indoor position environment. Among them, Wi-Fi and BLE used similar bandwidth of $2.4 \mathrm{GHz}$ radio wave frequency but based on $[14,25]$ BLE has more advantage than Wi-Fi because of lower material cost and high availability of BLE because of shorter channel width and 3 dedicated advertisement channel also supported by most modern smartphones which made BLE more advantageous than Wi-Fi technology.

BLE is introduced as a new form of wireless communication that is designed for short range communication [25-27]. BLE act as a replacement for cable to transmit the data into another device. Most of the time BLE stays in sleep mode constantly except when connection is initiated [15]. In the previous research most of the experiment on other literature are based on static user, where position of users are estimated while user are immobile. Which is why we will do experiment on dynamic user with fingerprinting algorithm and implement tracking element on the position by Extended Kalman Filter to see the difference.

In the Fingerprinting method we can get the object's location based on the signal strength that are channelled from the BLE, it consists of 2 main phase that are Offline and Online phase. Offline phase used to store the experimental condition of radio wave on a grid based possible location with signal strength received from each transmitter as the reading value, mean while online step is the phase where positioning be done where measurement of signal strength from each transmitter will be used to match the condition stored from previous step 
to determine the object's location. There are some methods proposed such as Machine Learning algorithm [17] to determine estimated position. The conclusion drawn on [17] is the method used to estimate position will affect the result of position estimation of an object.

Fingerprinting in indoor positioning systems was also known as scene analysis method which was used to measure the condition of radio frequency signal on the test field. The measurement of radio frequency was taken not only to determine position on the online phase but also to avoid wrong measurement because of obstacle material, human cause, etc. Much research has been focused on removing the noise caused by other factors, but there are no exact methods to remove it.

There are two main proposed methods in this paper to solve our problem. First one, this paper intended to implement a tracking algorithm based on Kalman filter but with the extended version because of the non-linearity of RSS measurement. We proposed this tracking algorithm because of the change from static user data collection to dynamic user data collection. Also, we proposed this filtering algorithm because in [13] we see an improved accuracy around 0.3 meter by using this filtering algorithm. The proposed method expected to improve the current accuracy by tracking the estimated position based on measured noise covariance, process noise covariance, and Kalman gain. Second, this paper intended to do the data capture dynamically because we want to see how much interference and is this factor will affect the experiment or not. We proposed to capture the data dynamically by walking in a route that we had created with some constraint and see whether the algorithm proposed could measure the distance or not.

\section{RELATED WORKS}

Indoor Positioning System using BLE technology has been experimented on many known techniques from Wi-Fi technology [7,8] like trilateration is a method of position estimation using the distance between mobile target and the receiver with the received RSSI as the measuring unit. The position of an object can be measured with a minimum of 3 estimated distance which is why this method was called trilateration. The disadvantages of this method is the simplicity of the measurement only based on the placement of BLE or Wi-Fi access point location and current RSS information which can change depending on environmental change also due to multiple interference on other radio signal that will cause the distance estimated contain some noises.

Due to the simplicity on basic idea of trilateration, many researcher $[7,8,10]$ has proposed a new idea to include other parameters that can be taken as measurement such as Time of Arrival (TOA) and Time Difference of Arrival (TDOA). TOA is the time measured for a radio wave from transmitter to reach receiver and TDOA is the measurement taken by examining the difference of time between each radio signal interval. The most accurate usage of TOA/TDOA has been proposed by [10] where radio wave propagation was modeled as probabilistic model and determination of position will be based on time-delay estimation algorithms and this method has proven to solve the fading effect of radio wave propagation.

To solve the problem of multipath radio wave phenomenon and more accurate measurement on positions, fingerprinting technique was developed. Fingerprinting on IPS uses Wi-Fi Access Point ID or BLE ID and RSSI where an experimental site with transmitter scattered then divides the space into small grids and collects the RSSI value on each grid to be saved into a database is called a radio map $[1,11]$. From here, there's two approaches that have been known so far to determine the position of receiver deterministic positioning algorithm and probabilistic positioning algorithm.

The deterministic positioning algorithm is based on the measured signal strength on radio map creation phase to match the real-time measured signal strength to determine the position of receiver. There are several deterministic positioning algorithms that has achieved goods result like K-Nearest Neighbour (KNN) and its variants which is one of the simplest algorithm that may utilize machine learning in estimate receiver position. The main idea of fingerprinting with $\mathrm{KNN}$ is to determine nearest position from measurement to the labelled position database and choose the $k$ number of nearest neighbor to calculate the estimated position by using distance estimation model like Manhattan distance or Euclidean distance. As the principle of fingerprinting is to match the measured RSS with radio map, it is similar to pattern recognition that has been the most commonly usage of machine learning where Artificial Neural Network (ANN) methods is used as learning factor algorithm in pattern recognition [29, 30]. Researcher in [17] has implemented ANN as fingerprint position method and the result has achieved a good pattern recognition method where the cumulative distribution function has a scalable model. Another more advanced deterministic technique that support machine learning as position estimation are Support Vector Machine (SVM) which use a supervised learning model to analyse linear or non-linear regression problem. The purpose is to analyse a regression model that can represent all the nearest data in the regression plane.

While deterministic positioning algorithm is based on signal that matches the corresponding fingerprint data to determine receiver position, probabilistic positioning algorithm approaches this by calculating the probability distribution of radio wave signal strength during a certain time and stored in fingerprint database to be used on online phase also during the online phase of positioning, the more static a user are on the position, the more accurate the result of position estimation will be which is why probabilistic positioning algorithms cannot be used to determine the position on a dynamic device. 
Table 1: Related Works Summary

\begin{tabular}{|c|c|c|c|c|}
\hline No & $\begin{array}{l}\text { Author } \\
\text { (s) }\end{array}$ & $\begin{array}{l}\text { Domain / } \\
\text { Variable }\end{array}$ & Method & Result \\
\hline 1 & {$[16]$} & $\begin{array}{l}\text { Lecturer } \\
\text { room, the area } \\
\text { of the test site } \\
\text { is about } 37.3 \\
\mathrm{~m} \text { by } 26.5 \mathrm{~m} \text {. } \\
3 \text { ibeacons } \\
\text { and } 3 \text { wifi } \\
\text { APs. }\end{array}$ & $\begin{array}{l}\text { Sigma-Point } \\
\text { Kalman Particle } \\
\text { Filter }\end{array}$ & $\begin{array}{l}\text { average } \\
\text { localization } \\
\text { error about } \\
10.37 \mathrm{~cm} \text { and } \\
71.63 \mathrm{~cm}\end{array}$ \\
\hline 2 & {$[3]$} & $\begin{array}{l}20 \text { BLE on } \\
2400 \mathrm{~m} 2 \\
\text { building size } \\
\text { tested on the } \\
\text { corridor }\end{array}$ & $\begin{array}{l}\text { Polynomial } \\
\text { Regression } \\
\text { Method }\end{array}$ & $\begin{array}{l}\text { Mean of Error } \\
1.84 \mathrm{~m}\end{array}$ \\
\hline 3 & [14] & $\begin{array}{l}2 \text { floor } \\
1200 \mathrm{~m}^{2} \text { each, } \\
81 \mathrm{BLE} \text { in } \\
\text { total }\end{array}$ & Ensemble Filter & $\begin{array}{l}\text { Accuracy of } \\
95.78 \%\end{array}$ \\
\hline 4 & [17] & $\begin{array}{l}4 \mathrm{BLE} \text { on } 4 \mathrm{~m} \\
\text { x } 6 \mathrm{~m} \text { room. }\end{array}$ & $\begin{array}{l}\text { Support Vector } \\
\text { Regression }\end{array}$ & $\begin{array}{l}\text { Mean of } \\
\text { Error } 134.92 \\
\mathrm{~cm}\end{array}$ \\
\hline 5 & [20] & $\begin{array}{l}\text { using WLAN } \\
\text { with one } \\
\text { access point }\end{array}$ & $\begin{array}{l}\text { Fingerprinting } \\
\text { Kalman Filter } \\
\text { and Position } \\
\text { Kalman Filter }\end{array}$ & $\begin{array}{l}\text { MRSE around } \\
5.8 \mathrm{~m}\end{array}$ \\
\hline
\end{tabular}

Author in [3] used Fingerprinting with Polynomial Regression Model (PRM) to calculate the standard deviation of errors for each BLE beacon. The main idea is to use the standard deviation of each BLE beacon to build the estimation of position with propagation model. First RSS received on real time was processed through fingerprinting for location and PRM to estimate the distance. Then, statistical methods from first Outlier Detection will be used to improve the distance estimation. The result of improved distance estimated will be processed through Extended Kalman Filter (EKF) to produce the estimated current location. The location estimated by EKF will be processed through second Outlier Detection then the output of this process will be used as the most appropriate location known. Result of the method proposed produced 1.84 $\mathrm{m}$ mean of error on localization which was good and based on the conclusion drawn the result has been boosted by dense deployment of BLE.

Other methods have proposed to filter the noise by using An Ensemble Filter Algorithm with Fingerprinting method in [14]. The research takes place in a two-floor retail store, and by using this filtering algorithm they achieve 2 meters accuracy which is good considering many noise around the store such as shoppers, staff moving, product and many more. It is stated that these methods achieve around $95.78 \%$ accuracy.
In [16] the researcher used an Improved Kalman Filter Algorithm (Sigma-Point Kalman Particle Filtering) to solve the Indoor Positioning problem in a Lecturer room. The Author said it is an improved version that improves Kalman Filter by leveraging the unscented transformation of UKF and the weighting method of PF. These methods achieve accuracy up to $20.1 \%$ higher than Kalman Filter, and error rate around $10.37 \mathrm{~cm}$ and $71.63 \mathrm{~cm}$. and in [20] the author use a Fingerprinting Kalman Filter and compare it with Positioning Kalman Filter and using WLAN with one access point as the broadcaster, the result is improved but we can see that the error rate is still high around 5.8 meter.

Machine learning has also been used in IPS development, researcher on [17] tested 4 machine learning regression method: ANN, Multiple Linear Network (MLN), Random Forest Regression (RF) and Support Vector Regression (SVR four of them tested on the same dataset of room size $4 \mathrm{~m} \times 6 \mathrm{~m}$ with 4 beacon placed. Conclusion drawn by the researcher is that SVR produces the best performance.

Based on the literature review of the Table 1, BLE is the technology with the biggest advantage to be used as Indoor Positioning System. The most advantageous area of BLE technology are BLE has shorter channel width and total of 40 channel which mean it has higher speed on receiving signal also the low energy consumption according to [28] that allow BLE to be used for several month at least with the most draining configuration used. State-of-the-art method used based on the literature review is fingerprinting method. This method has been proven solve the fading effect of radio wave signal and removed the error of estimation caused by multipath effect.

On this paper, we developed an indoor positioning system using BLE as radio wave signal transmitter and smartphone as the receiver of Bluetooth radio wave signal. As for the fingerprinting method we will use weighted sum model as position estimation and EKF to implement tracking by tracking position method. We proposed Weighted Sum as a position method because it is a simple positioning method and yet give a precise result in positioning [17], as for Extended Kalman Filter we apply it as our tracking algorithm as we have study from many source that it is a common filter used in Positioning scheme and the result is good for noise filtering also it's commonly used on GPS tracking system when navigation on underpass or other not satellite zone. In this collection data method, we collect the data dynamically to see whether the algorithm that we propose would filter the noise or not. As we know in many research before their collection data is done statically. 


\section{PROPOSED METHODS}

\subsection{Fingerprinting}

In this paper we proposed an Indoor Positioning System by using BLE with fingerprinting method. There are two main phases of fingerprinting method proposed in this paper shown in Figure 1 offline phase is designed for learning the RSS at each reference point (Figure 2). This method simply creates Radio Map Database by gathering on each of the reference points and started receiving RSS from all BLE beacon then send the representative data to database. One row of data on the radiomap database consisting $2 \mathrm{~d}$ coordinate value $(\mathrm{x}, \mathrm{y})$ where the Reference point (gathered point) are and each BLE beacon RSS received. The main task of this phase is to collect fingerprint data to build a model [5].

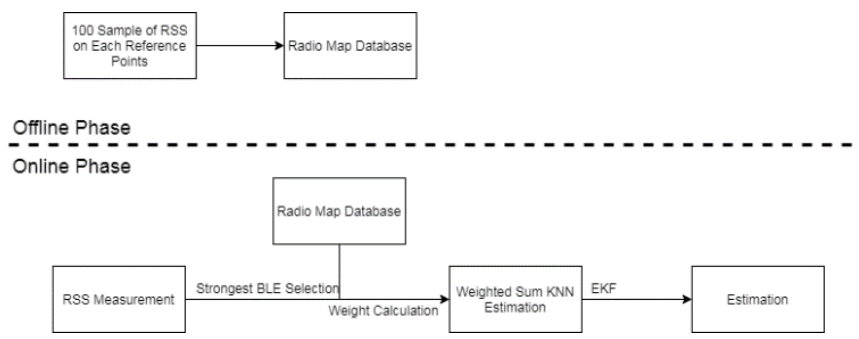

Figure 1: Phase of Localization

In the Online phase, the measurements are taken based on every each route. The position will be estimated using localization algorithm and will be compared with the data that is already stored in the database. In our research the sample will be taken with interval of 5 seconds. Then the sample data containing RSS value will be tracked by using Extended Kalman Filter and Weighted Sum that will measured with Euclidean distance and then ranked using the K-nearest Neighbor, resulting a single value consisting of their coordinate $(\mathrm{x}, \mathrm{y})$

\subsection{Weighted Sum}

Weighted Sum is proposed because it is ease of use and it is the simplest and popular method to rank a dataset [24]. Weighted Sum model was used to estimate the position from the measured RSS. In this research, weighted sum consists of the following five steps:

1. Calculate the distance between the measured RSS and the Reference Points RSS using Euclidean distance

$$
D(m p, r p)=\sqrt{\sum_{i=23}^{23}\left(R S S_{i}^{m p}-R S S_{i}^{r p}\right)}
$$

2. Sort the distance in ascending order

3. Select $k$ number of $R P$ with the smallest distance from the measured RSS

4. Calculate the weight of each reference points by using (2)

$$
W_{i}=\frac{D_{i}}{\sum_{i=k}^{k} D_{i}}
$$

5. Calculate the predicted $x$ and $y$ coordinate using (3)

$$
\hat{x}=\sum_{i=k}^{k}\left(W_{i} x_{i}^{r p}\right) \text { and } \hat{y}=\sum_{i=k}^{k}\left(W_{i} y_{i}^{r p}\right)
$$

Where $\hat{x}$ is the estimated $x$ position and $\hat{y}$ is the estimated $y$ position. $x_{i}^{r p}$ is from $x$ reference point value on the $i^{\text {th }}$ nearest $R P$ and $y_{i}^{r p}$ is from the $y$ reference point value on the $i^{\text {th }} R P$.

\subsection{Extended Kalman Filter}

Extended Kalman Filter is a more advanced form of Kalman Filter. The main difference between both methods was Kalman Filter is designed to handle linear problem [22, 28]. But in real life, most problems are nonlinear and whenever we use basic form of Kalman Filter to solve nonlinear problem the performance will degrade. That is why extended form of Kalman Filter or EKF is designed to specifically handle nonlinear problem.

Based on [3, 14-18] the propagation of radio wave signal on BLE technology and Wi-Fi technology are exactly linear. The nonlinearity of BLE radio wave propagation is proved by increasing the distance between receiver and transmitter but the RSS received result is not sequential as sometimes the result may duplicate because of various noise [6] on different distance. This is why we intend to implement EKF as a tracking solution to our problem.

Estimation of location from Fingerprinting method is inputted to EKF in our proposed algorithm track receiver current location by fusing current and historical information. The state vector of EKF in this paper is

$$
X=\left[\begin{array}{c}
x \\
v x \\
y \\
v y
\end{array}\right]
$$

EKF lifecycle is basically the same as Kalman Filter where it consists of prediction of state and update parameter based on last prediction. Model of state vector and covariance matrix used in our proposed method are represented in (6)

$$
\begin{gathered}
x_{k+1 \mid k}=\varphi_{k, k+1}+x_{k \mid k} \\
P_{k+1 \mid k}=\varphi_{k, k+1} P_{k \mid k} \varphi_{k, k+1}^{T}+Q_{k}
\end{gathered}
$$

Where $x_{k+1 \mid k}$ is the state vector, $x_{k \mid k}$ is the previous state vector at epoch $k, Q_{k}$ is standard deviation of process noise and $\varphi_{k, k+1}$ is a $4 \times 4$ transition matrix and the constant value will be (6)

$$
\varphi_{k, k+1}=\left[\begin{array}{cccc}
1 & \Delta t & 0 & 0 \\
0 & 1 & 0 & 0 \\
0 & 0 & 1 & \Delta t \\
0 & 0 & 0 & 1
\end{array}\right]
$$

Where $\Delta t$ used is the difference between two epochs.

$$
\boldsymbol{Z}_{k}=\boldsymbol{H}_{k} x_{k \mid k}+v_{k}
$$

Equation (7) represent the measurement model proposed where $\boldsymbol{Z}_{k}$ is the measurement and $v_{k}$ is the vector of velocity at the time of $k$. 
Kalman update of parameter are used to update state vector and covariance matrix as shown in (8)

$$
\begin{gathered}
\boldsymbol{K}_{k}=P_{k \mid k-1} \boldsymbol{H}_{k}^{T}\left(\boldsymbol{H}_{k} P_{k \mid k-1} \boldsymbol{H}_{k}^{T}+\boldsymbol{R}_{k}\right)^{-1} \\
x_{k \mid k}=x_{k \mid k-1}+\boldsymbol{K}_{k}\left(\boldsymbol{Z}_{k}-\boldsymbol{H}_{k} x_{k \mid k-1}\right) \\
P_{k \mid k}=\left(I-\boldsymbol{K}_{k} \boldsymbol{H}_{k}\right) P_{k \mid k-1}\left(I-\boldsymbol{K}_{k} \boldsymbol{H}_{k}\right)^{T}+\boldsymbol{R}_{k}
\end{gathered}
$$

Where $\boldsymbol{K}_{k}$ is the kalman gain and $\boldsymbol{H}_{k}$ is an identity matrix, $\boldsymbol{R}_{k}$ is the measurement error covariance matrix at time of $k$ and $P_{k \mid k}$ is the estimate error covariance.

The result of EKF will be evaluated using Root Mean Square Error (RMSE) where the estimated position will be subtracted by ground truth position then calculate the result by square root the value.

\section{EXPERIMENTAL DESIGN}

First, we configured 23 BLE beacon from Nordic Semiconductor nRF51822 Bluetooth Smart Beacon that is shown in Figure 3 with transmit power of $0 \mathrm{dBm}$ and transmit interval of 100 milliseconds. Then the beacon will be deployed in this research as shown in Figure 2 represented by the blue dots as beacon deployed where on the site will be placed at $1.2 \mathrm{~m}$ height from the floor.

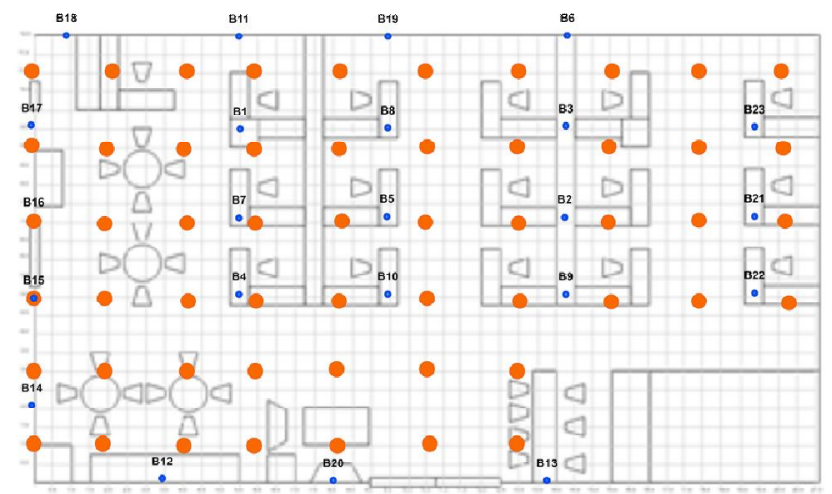

Figure 2: Testbed

The test field is built on the 8th floor of the Bina Nusantara University Kampus Anggrek and the device used for Bluetooth receiver is a smartphone device with specifications

\begin{tabular}{|c|c|}
\hline \multicolumn{2}{|r|}{ Xiaomi Redmi Note 8} \\
\hline os & Android 9.0 (Pie); MIUI 11 \\
\hline Chipset & Qualcomm SDM665 Snapdragon $665(11 \mathrm{~nm})$ \\
\hline CPU & Octa-core (4×2.0 GHz Kryo 260 Gold \& 4x1.8 GHz Kryo 260 Silver) \\
\hline GPU & Adreno 610 \\
\hline WLAN & Wi-Fi $802.11 \mathrm{a} / \mathrm{b} / \mathrm{g} / \mathrm{n} / \mathrm{ac}$, dual-band, Wi-Fi Direct, hotspot \\
\hline Bluetooth & $4.2, \mathrm{~A} 2 \mathrm{DP}, \mathrm{LE}$ \\
\hline GPS & Yes, with A-GPS, GLONASS, BDS \\
\hline
\end{tabular}
of "Xiaomi Redmi note 8" shown on Table 2.

Table 2: Device Specification

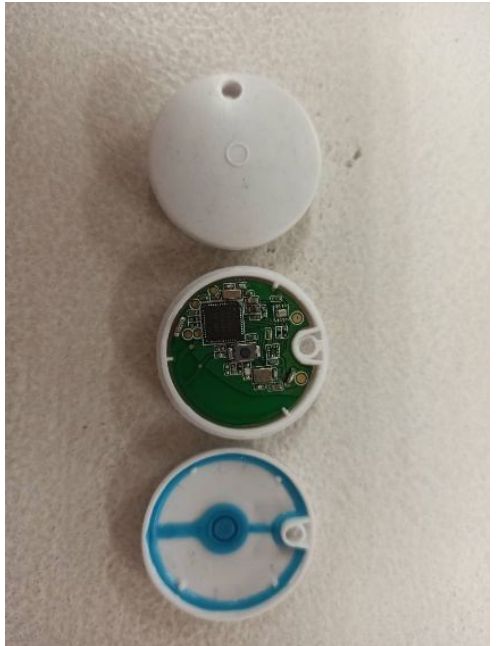

Figure 3: BLE beacons

Test field used in this experimental design is an office room with size $12 \mathrm{~m}$ x $19 \mathrm{~m}$ shown at Figure 5. Reference point are placed with 2 meters gap each shown in Figure 4 and total of 54 reference point are used in the radio map. On each reference point 100 samples were taken by smartphone device specified on Table 2 to log the RSS received while standing on the point around 3 minutes for the entire sample of a point to be taken.

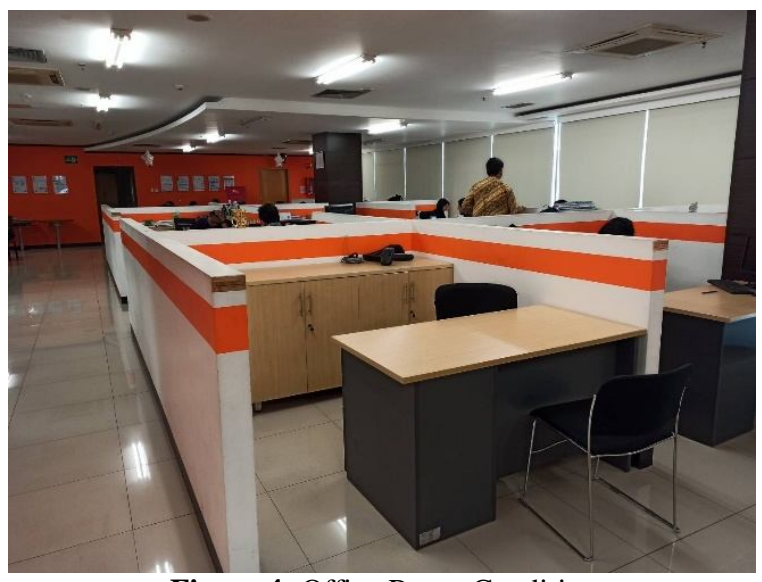

Figure 4: Office Room Condition

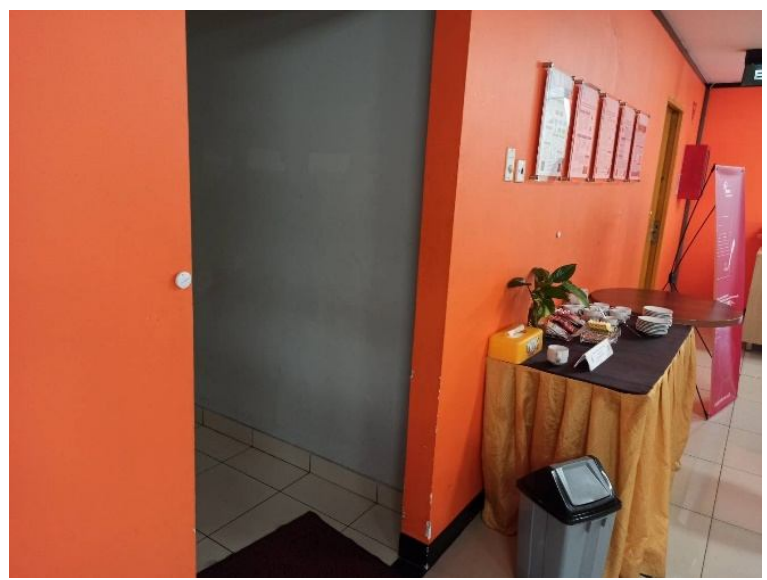

Figure 5: Bluetooth Placement in Room 
The Application needs to wait for all of the beacon to respond in order to start the scan. After all the beacon transmit the signal, the scan will start repeatedly until it reaches the maximum number of the samples which is 100 sample per $R P$. To avoid zero values and repetitive values we add $1 \mathrm{~s}$ interval per scan. The result will be used as a matching data for the reference point and this will determine the distance of each testing route.

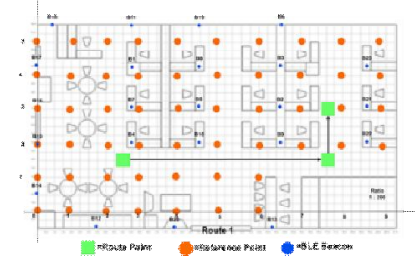

(Route 1)

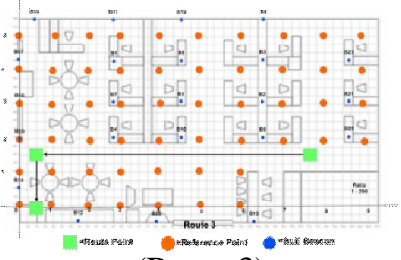

(Route 3)

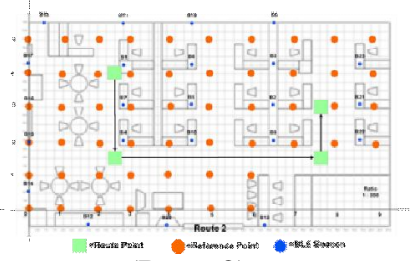

(Route2)

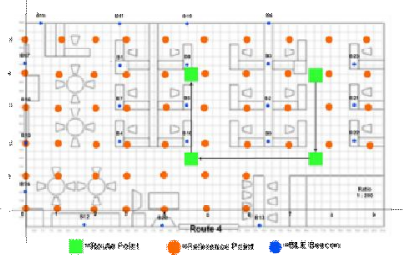

(Route 4)

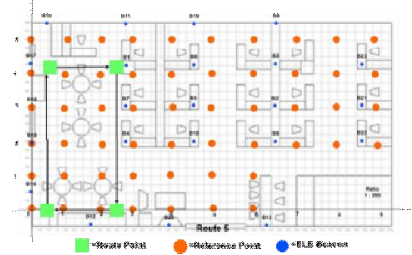

(Route 5)

Figure 6: Route taken in this experiment

Testing routes are defined randomly in between the reference points, but with some constraint that the route should be inside the reference point to maximize the data sampled. If the route is outside the reference point, there is a possibility that the signal frequency will not be delivered correctly. We designed the route above to get all the possibilities in the room, so we designed it in many different approaches. For each route we gather around 4 or 5 times data to see the RSS data sampled and compare it, we make sure that each time we collect the data, the velocity of pedestrian movement are stable, to reduce the probability of being too slow or too fast. We also take notes which data that the pedestrian is turning into left or right to make sure the timestamp of the data is correct. All Route Experiments in this paper are taken with $5 \mathrm{~s}$ scan interval and constant velocity of $20 \mathrm{~cm} / \mathrm{s}$.
Then the data for each route are processed by using the Weighted Sum to estimate the location of the pedestrian per each row data, and then inputted to EKF to create a more precise track measurement to ground truth. Parameters used in this experiment are $k$ (number of nearest $R P$ ) is 5 on weighted sum method, $Q$ (process noise) is 1 processed with $R$ (measurement error or noise) 1.

\section{RESULT \& DISCUSSION}

The experiment was conducted with 5 different routes (Figure 4) and per route is tested around 3-5 minutes with around 20-50 row of data and each data will be taken repeatedly per 5 seconds to avoid data loss ( 0 value for a beacon) and with each route being selected with some constraint. For each route assuming we walk around the route with the same velocity, the data of each route then proceeded to be processed by Weighted Sum.

Route 1

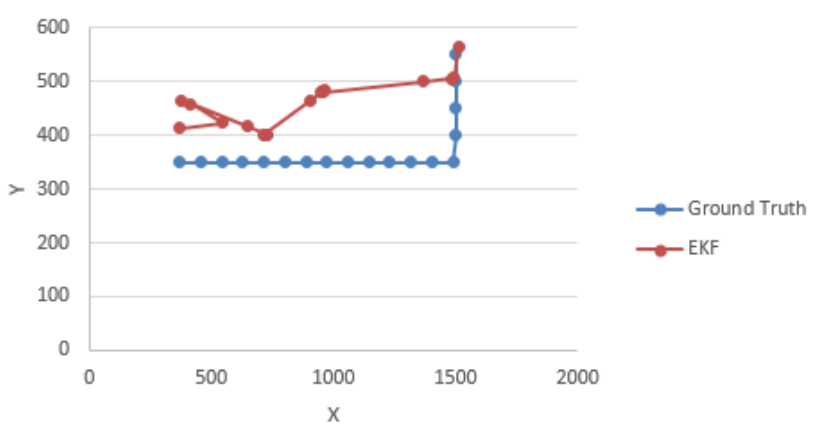

(Route 1)

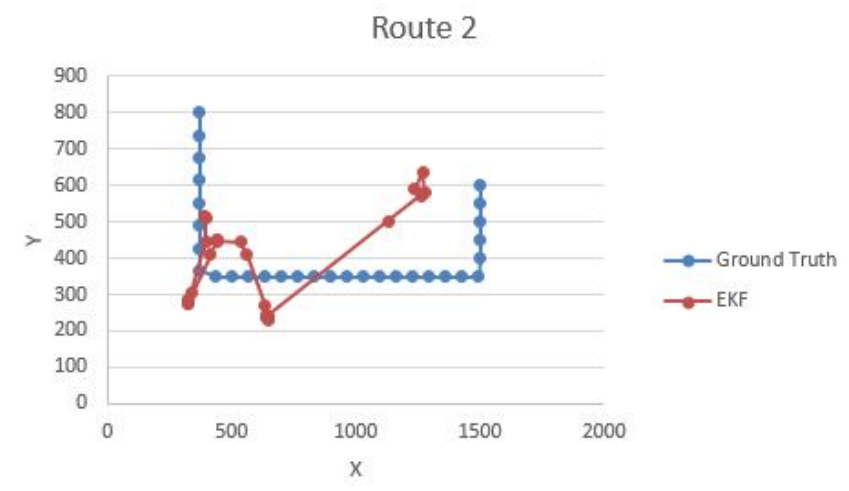

(Route 2) 


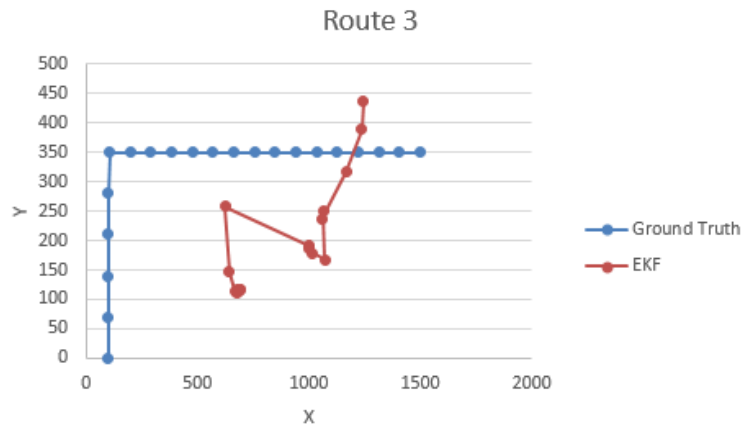

(Route 3)

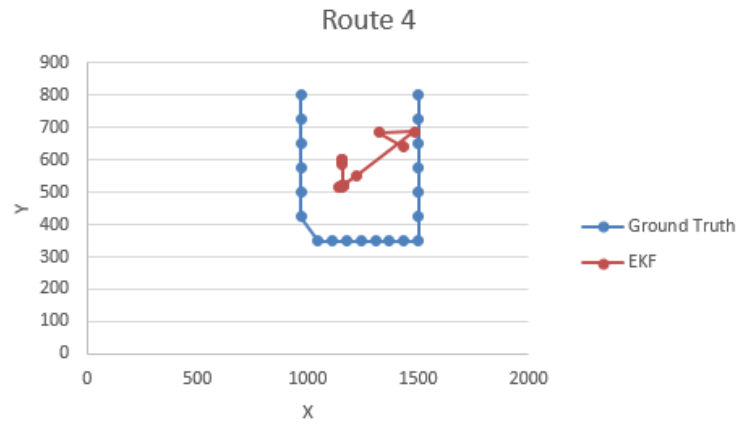

(Route 4)

Route 5

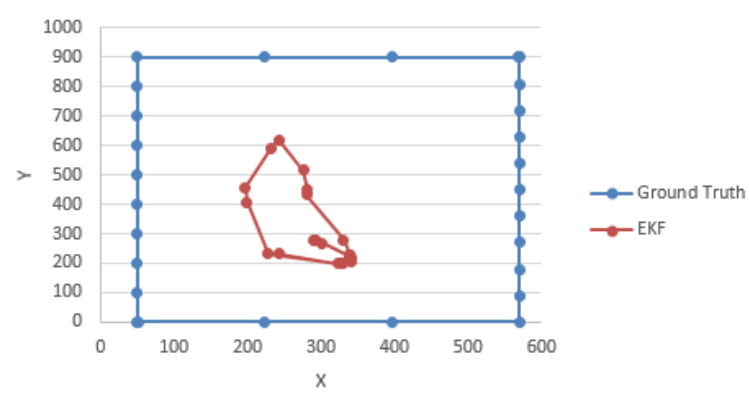

(Route 5)

Figure 7: Graph Result

Table 3: Performance Summary

\begin{tabular}{|l|l|c|c|c|c|c|}
\hline \multirow{2}{*}{ Route Taken and Method } & \multicolumn{5}{|c|}{ PERFORMANCE } \\
\cline { 3 - 7 } & $\begin{array}{c}\text { Mean of Error } \\
(\mathrm{cm})\end{array}$ & $\begin{array}{c}\text { Min of Error } \\
(\mathrm{cm})\end{array}$ & $\begin{array}{c}\text { Max of Error } \\
(\mathrm{cm})\end{array}$ & $\begin{array}{c}\text { Median of Error } \\
(\mathrm{cm})\end{array}$ & $\begin{array}{c}\text { 90th Percentile } \\
(\mathrm{cm})\end{array}$ \\
\hline Route 1 & $\begin{array}{l}\text { Weighted Sum + } \\
\text { EKF }\end{array}$ & 210.73 & 9.10 & 554.39 & 166.27 & 406.41 \\
\hline Route 2 & $\begin{array}{l}\text { Weighted Sum + } \\
\text { EKF }\end{array}$ & 341.88 & 94.39 & 856.87 & 276.24 & 667.31 \\
\hline Route 3 & $\begin{array}{l}\text { Weighted Sum + } \\
\text { EKF }\end{array}$ & 353.06 & 99.46 & 627.58 & 291.75 & 599.29 \\
\hline Route 4 & $\begin{array}{l}\text { Weighted Sum + } \\
\text { EKF }\end{array}$ & 242.19 & 40.71 & 389.88 & 232.48 & 351.98 \\
\hline Route 5 & $\begin{array}{l}\text { Weighted Sum + } \\
\text { EKF }\end{array}$ & 364.22 & 175.18 & 722.89 & 301.15 & 592.08 \\
\hline
\end{tabular}

The graph in Figure 7 shows the difference between ground truth and EKF method. The distance between ground truth and EKF method are the Error. Big differences are shown compared to other research because we take the RSS measurement while moving and many working people on the experimental site that may cause the effect of the human body composed of $70 \%$ water and can absorb the radio frequency signal of $2.4 \mathrm{GHz}$ [12]. But, based on Table 3 our 
fingerprinting method achieved mean of error at $2.13 \mathrm{~m}$ to $3.16 \mathrm{~m}$ on our experimental site with size $12 \mathrm{~m} \times 19 \mathrm{~m}$ with other material, obstacle and human activity included also the grid size on $2 \mathrm{~m}$ between each $R P$ is considered a big improvement compared to other technology with same experimental design. For example, in [12] with results of 8.68 $\mathrm{m}$ without human body interference and $9.82 \mathrm{~m}$ with human body interference even though the measurement was taken by taking measurement on each point, our method has more error to be measured in and produced a more accurate position estimation.

The performance of EKF from our experimental design with $\mathrm{Q}$ set as 1 and $\mathrm{R}$ set as 1 achieved mean of error at $2.13 \mathrm{~m}$ to $3.16 \mathrm{~m}$ one of the reason for inadequacy of movement in the route shown by EKF is because of the position detection by smartphone has many redundant position which change the calculation of Kalman gain and made the movement harder to track.

\section{CONCLUSION AND FUTURE WORKS}

We have proposed a positioning method for tracking people in motion in indoor environment by adding Extended Kalman Filter to address the issue of tracking by tracking where user is moving dynamically. The purpose of EKF was to track the path taken based on measurement, covariance and process noise. The proposed method achieves an average mean of error around $302,42 \mathrm{~cm}$. We also test our proposed methods by testing in a real-life case when the user is walking. we observe that there is some time taken to transmit the signal to the receiver that will cause some RSS to contain some noises and if compared to the static data the RSS in dynamic data contain much more noises. But as we analyse the data the Extended Kalman Filter didn't really do much as we expected. This is due to our data still contain some noise. After the prediction using Weighted Sum our data is far from the ground truth, so the result of our EKF will also be far from it.

In the next study, finding a new way to change traditional ways of capturing fingerprint data is an interesting topic. As traditional ways of capturing fingerprint data require time and labour intensive also an expert in capturing the data. Larger the size of the positioning environment requires more time. Thus, it is necessary to find new ways of obtaining fingerprint data quickly and easily. Also, it is necessary to find the best filtering algorithm that can be best applied into each environment. In [21] we see that a drift removal algorithm could be used to get better results before location estimation. We think that this will be another solution to get better results.

\section{ACKNOWLEDGMENT}

We would like to acknowledge the support given by BINUS Graduate Program and Bina Nusantara University for giving us a facility to collect the data sample.

\section{CONFLICT OF INTERESTS}

The authors declare that there is no conflict of interests regarding the publication of this paper.

\section{REFERENCES}

1. Zhao, Y., Liu, C., Mihaylova, L. S., \& Gunnarsson, F. Gaussian Processes for RSS Fingerprints Construction in Indoor Localization, in 21 st International Conference on Information Fusion, FUSION, 2018.

https://doi.org/10.23919/ICIF.2018.8455842

2. Fan, G. F., Guo, Y. H., Zheng, J. M., \& Hong, W. C. Application of the weighted k-nearest neighbor algorithm for short-term load forecasting, in Energies, 12(5), 2019.

3. Zhuang, Y., Yang, J., Li, Y., Qi, L., \& El-Sheimy, N. Smartphone-based indoor localization with bluetooth low energy beacons, in Sensors, 16(5), 596, 2016.

4. Brena, R. F., García-Vázquez, J. P., Galván-Tejada, C. E., Muñoz-Rodriguez, D., Vargas-Rosales, C., \& Fangmeyer, J. Evolution of Indoor Positioning Technologies: A Survey, in Journal of Sensors, 1-21, 2017.

5. Xia, S., Liu, Y., Yuan, G., Zhu, M., \& Wang, Z. Indoor fingerprint positioning based on Wi-Fi:An overview, in ISPRS International Journal of Geo-Information, 6(5) , 2017.

6. Akram, M. A., Liu, P., Tahir, M. O., Ali, W., \& Wang, Y. A state optimization model based on kalman filtering and robust estimation theory for fusion of multi-source information in highly non-linear systems, in Sensors, 19(7), 1687, 2019. https://doi.org/10.3390/s19071687

7. Zegeye, W. K., Amsalu, S. B., Astatke, Y., \& Moazzami, F. WiFi RSS fingerprinting indoor localization for mobile devices, in 2016 IEEE 7th Annual Ubiquitous Computing, Electronics \& Mobile Communication Conference (UEMCON), 2016.

8. Draghici, I.-C., Vasilateanu, A., Goga, N., Pavaloiu, B., Guta, L., Mihailescu, M. N., \& Boiangiu, C.-A. Indoor positioning system for location based healthcare using trilateration with corrections, in 2017 International Conference on Engineering, Technology and Innovation (ICE/ITMC), 2017.

9. Jianyong, Z., Haiyong, L., Zili, C., \& Zhaohui, L. RSSI based Bluetooth low energy indoor positioning, in 2014 International Conference on Indoor Positioning and Indoor Navigation (IPIN), 2014.

10. Keunecke, K., \& Scholl, G. Deriving 2D TOA/TDOA IEEE $802.11 \mathrm{~g} / \mathrm{n} / \mathrm{ac}$ location accuracy from an experimentally verified fading channel model, in International Conference on Indoor Positioning and Indoor Navigation, 2013.

11. Son S, Park Y, Kim B, Baek Y. Wi-Fi fingerprint location estimation system based on reliability, in $J$ Korean Inst Commun Inf Sci 38(6):531-539, 2013.

12. Garcia-Villalonga, S., \& Perez-Navarro, A. Influence of human absorption of $\mathrm{Wi}-\mathrm{Fi}$ signal in indoor positioning with Wi-Fi fingerprinting, in 2015 
International Conference on Indoor Positioning and Indoor Navigation (IPIN), 2015.

13. Lee, S. H., Lim, I. K., \& Lee, J. K. Method for Improving Indoor Positioning Accuracy Using Extended Kalman Filter, in Mobile Information Systems, 2016.

14. Frost, C., Jensen, C. S., Luckow, K. S., Thomsen, B., \& Hansen, R. Bluetooth indoor positioning system using fingerprinting, in Lecture Notes of the Institute for Computer Sciences, Social-Informatics and Telecommunications Engineering, 81 LNICST, 136-150, 2012.

15. Tosi, J., Taffoni, F., Santacatterina, M., Sannino, R., \& Formica, D. Performance Evaluation of Bluetooth Low Energy: A Systematic Review, in Sensors (Basel, Switzerland), 17(12), 2898, 2017.

16. Sung, K., Lee, D. K. R., \& Kim, H. Indoor pedestrian localization using ibeacon and improved kalman filter, in Sensors (Switzerland), 18(6), 2018.

https://doi.org/10.3390/s18061722

17. Alexander, Ivan \& Kusuma, Gede. Predicting Indoor Position Using Bluetooth Low Energy And Machine Learning, in International Journal of Scientific \& Technology Research. 8. 1661-1667, 2019.

18. Hossain, A. K. M. M., \& Soh, W. S. A survey of calibration-free indoor positioning systems, in Computer Communications, 2015.

19. Davidson, P., \& Piché, R. A Survey of Selected Indoor Positioning Methods for Smartphones, in IEEE Communications Surveys and Tutorials, 2017.

20. Ali-Löytty, S., Tommi Perälä, Honkavirta, V., \& Piché, R. Fingerprint Kalman Filter in indoor positioning applications, in Proceedings of the IEEE International Conference on Control Applications, 2009.

21. Huang, C. H., Lee, L. H., Ho, C. C., Wu, L. L., \& Lai, Z. H. Real-time RFID indoor positioning system based on kalman-filter drift removal and heron-bilateration location estimation, in IEEE Transactions on Instrumentation and Measurement, 2015.

22. Rodney Faragher, Understanding the Basis of the Kalman Filter Via a Simple and Intuitive Derivation, in IEEE Signal Processing Magazine, 2012.

23. Jang, B., \& Kim, H. Indoor positioning technologies without offline fingerprinting map: A survey, in IEEE Communications Surveys and Tutorials, 2019.

24. Marler, R. T., \& Arora, J. S. The weighted sum method for multi-objective optimization: New insights, in Structural and Multidisciplinary Optimization, 2010.

25. Gomez, Carles \& Oller Bosch, Joaquim \& Paradells, Josep. Overview and Evaluation of Bluetooth Low Energy: An Emerging Low-Power Wireless Technology, in Sensors (Basel, Switzerland). 12. 11734-53. 10.3390/s120911734, 2012.

26. G. Patti, L. Leonardi and L. Lo Bello. "'A Bluetooth Low Energy real-time protocol for Industrial Wireless mesh Networks, in IECON 2016 - 42nd Annual Conference of the IEEE Industrial Electronics Society (Florence), 2016.

27. Darroudi, S. M., \& Gomez, C. Bluetooth Low Energy Mesh Networks: A Survey, in Sensors (Basel, Switzerland), 17(7), 1467, 2017.
28. Jahan, K., Engineering, C., Lakshmaiah, K., Foundation, E., \& Pradesh, A. International Journal of Emerging Trends in Engineering Research Comparison of MGBEKF and UKF Algorithms for Bearings-Only Tracking, in International Journal of Emerging Trends in Engineering Research 7(7), 44-47, 2019. https://doi.org/10.30534/ijeter/2019/02772019

29. Don, A., Africa, M., Journal, I., Africa, A. D. M., Naco, I. K. A., Castillo, J. J. M., ... Wu, S. R. T. International Journal of Emerging Trends in Engineering Research ANN Distance Protection for Transmission Lines, in International Journal of Emerging Trends in Engineering Research, 2019. https://doi.org/10.30534/ijeter/2019/07782019

30. Sasi Bhanu, J., Baswaraj, D., Bigul, S. D., \& Sastry, J. K. R. Generating test cases for testing embedded systems using combinatorial techniques and neural networks based learning model, in International Journal of Emerging Trends in Engineering Research, 7(11), 417-42, 2019. https://doi.org/10.30534/ijeter/2019/047112019 